\title{
Case Study of Empirical Beam Hardening Correction Methods for Dimensional X-ray Computed Tomography Using a Dedicated Multi-material Reference Standard
}

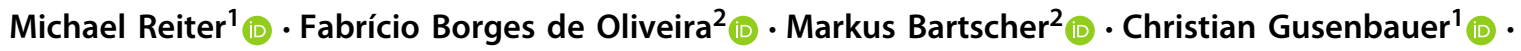 \\ Johann Kastner ${ }^{1}$
}

Received: 22 December 2017 / Accepted: 4 December 2018 / Published online: 12 December 2018

(c) The Author(s) 2018

\begin{abstract}
This paper presents a case study of two selected beam hardening correction methods and their effects on dimensional measurements of multi-material objects. The methods under test are empirical cupping correction (ECC) and empirical dual energy calibration (EDEC). These methods were originally developed for medical applications and their potential for the reduction of artefacts is typically only analysed based on grey value images. For testing and benchmarking of the mentioned methods for dimensional metrology, a dedicated multi-material reference standard—a multi-material hole cube-is used. This reference standard was originally developed for acceptance testing of CT systems. This paper shows a second application of this standard. The reference standard has been calibrated by tactile measurements to assess centre-centre distance errors as well as patch-based bidirectional length measurement errors on beam hardening corrected data and on uncorrected data. For the application of the method also to industrial multi-material scenarios, slight modifications of the ECC method are proposed. Practical aspects of both the ECC and the EDEC approaches as well as measurement results are analysed and discussed in detail. ECC was able to significantly improve dimensional measurements and was especially able to reduce extreme errors occurring in particular in multi-material scenarios by a factor of more than 4. EDEC, the dual-energy approach, reduced grey value inhomogeneities caused by artefacts even more. Its performance for dimensional measurements was however a little worse than ECC. EDEC data resulted in a slightly larger total range of residual measurement errors, mainly due to an elevated noise level.
\end{abstract}

Keywords Industrial computed tomography $\cdot$ Dimensional metrology $\cdot$ Multi-material measurements $\cdot$ Beam hardening correction - Material influence

\section{Introduction}

Michael Reiter

michael.reiter@fh-wels.at

Fabrício Borges de Oliveira

fabricio.borges@ptb.de

Markus Bartscher

markus.bartscher@ptb.de

Christian Gusenbauer

christian.gusenbauer@fh-wels.at

Johann Kastner

johann.kastner@fh-wels.at

1 University of Applied Sciences Upper Austria - Wels Campus, Stelzhamerstraße 23, 4600 Wels, Austria

2 Physikalisch-Technische Bundesanstalt (PTB), Bundesallee 100, 38116 Brunswick, Germany
The use of X-ray computed tomography (XCT) devices as a coordinate measurement system (CMS) has made the complex task of dimensional measuring multi-material objects possible and also allows the measurement of assemblies in a mounted state. However, the reliable metrology of multimaterial components with XCT is a challenging task, since non-linearities in the projection data, caused by e.g. beam hardening and scattered radiation, lead to severe artefacts (i.e. imaging errors) in the reconstructed XCT images. These artefacts can severely hinder the performance of reliable dimensional measurements.

Several methods to correct the non-linear behaviour of multi-material components have been published. However, the potential for improvement is typically depicted only 
by grey-value-based image analyses and deducing quality metrics rather than evaluating the influence on material boundaries (surfaces) or errors of feature-based dimensional measurements [1].

This paper presents a comprehensive case study of the metrological performance of multi-material measurements of two selected beam hardening correction (BHC) approaches. Furthermore, this work attempts to perform a critical analysis of these methods from a metrological point of view by applying a calibrated multi-material reference standard for testing and benchmarking the different approaches. This reference standard - a multi-material hole cube (MMHC) [2] — was originally developed for the acceptance testing of XCT-based CMSs. A second application of this standard is shown in this paper.

\section{Case Study}

\subsection{Beam Hardening Correction Methods for Multi-material Parts}

The study uses Empirical Cupping Correction (ECC) described by Kachelrieß et al. [3] and Empirical Dual Energy Calibration (EDEC) described by Kachelrieß et al. $[4,5]$ that correct non-linearities in the projection data due to the polychromatic nature of X-rays and partially scattered radiation. Contrary to other correction approaches for beam hardening artefacts [6-9], ECC and EDEC do not rely on iterative forward projections and filtered-back projections and they do not need any preliminary knowledge about the specimen (geometry, position and materials) and the spectra in use. This is - in principle - a massive advantage for the user of these methods and makes it worth quantifying whether the methods can be proven to provide an advantage for dimensional measurements also for the case of multi-material measurements. ECC and EDEC are practical methods with great potential for metrology too, but so far there have been no qualification studies on these applications. More complex methods with a high calculation burden that rely on iterative reconstructions [10-12] or on special data fusion techniques [13] are currently not practical for industrial applications and are therefore out of the scope of this work.

The ECC method was originally designed to correct linearity issues for specimens made of one material only. With $q_{1}(D)$ being the log-attenuation values, the ECC method models the relation to the line of integration $D$ by the correction function $P\left(q_{1}\right)$. The index of $q_{1}$ is only of importance in the context of EDEC, the dual-energy approach, where an additional $q_{2}$ is used. In [3] and within this work, $P\left(q_{1}\right)$ is limited to linear combinations of $P_{n}\left(q_{1}\right)$, which leads to corrected attenuation values $p_{\text {ECC }}$ approximated by a polynomial function of the order of $N$

$$
\begin{aligned}
p_{\mathrm{ECC}} & =P\left(q_{1}\right)=\sum_{n=0}^{N} c_{n} P_{n}\left(q_{1}\right) \\
& =\sum_{n=0}^{N} c_{n} q_{1}^{n}=c_{1} q_{1}+c_{2} q_{1}^{2}+c_{3} q_{1}^{3}+\cdots \text { with } c_{0}=0 .
\end{aligned}
$$

The linearity of the Radon transform $R$ is used to calculate $N$ basis images

$f_{n}(\mathbf{r})=R^{-1} q_{1}^{n}$

that are combined to the reconstructed and corrected XCT image

$f(\mathbf{r})=\sum_{n=0}^{N} c_{n} f_{n}(\mathbf{r})$

by a vector of coefficients $c$ that minimise the deviation between $f(\mathbf{r})$ and a given template image $t(\mathbf{r})$, with $\boldsymbol{r}$ being the location in the corrected XCT image. This method assumes that the specimen is made of homogeneous material and, for example, does not have any kind of density gradient. In other words, the homogeneity of attenuation coefficients in the corrected XCT image is maximised and as a consequence, artefacts caused by beam hardening and scattered radiation are minimised. Although preliminary knowledge of the specimen is not required, it can be beneficial to use preliminary or expert knowledge for the generation of the template image (for further discussion see Sect. 3.1). The ECC optimisation procedure proposed by Kachelrieß et al. determines coefficients $\mathbf{c}$ by the optimisation metric

$E=\min _{\mathbf{c}} \sum_{\mathbf{r}} w(\mathbf{r})(f(\mathbf{r})-t(\mathbf{r}))^{2}$,

where $w(\mathbf{r})$ is a weight image that can be used to emphasise or exclude regions from the optimisation, e.g. exclude regions that are affected by partial volume effects [3]. Although ECC was initially proposed for medical applications, it can be applied to industrial parts made of one homogeneous material [1]. For industrial parts made of multi-material components, it is proposed to generalise the template $t$ to

$t(\mathbf{r})=\left\{\begin{array}{r}\mu_{M} \text { for } \mathbf{r} \in \text { material } M, \\ \ldots \\ \mu_{2} \text { for } \mathbf{r} \in \text { material } 2, \\ \mu_{1} \text { for } \mathbf{r} \in \text { material } 1, \\ \mu_{0}=0 \text { for } \mathbf{r} \in \text { air, }\end{array}\right.$

that contains attenuation coefficients $\mu$ for $M$ different materials including air. Furthermore, the weight $w(\mathbf{r})$ is set to zero in regions where the correspondence to a material is uncertain or if a region is not of interest. 
$w(\mathbf{r})=\left\{\begin{array}{l}>0 \text { for } \mathbf{r} \in \text { materials } 1 \ldots M \text { and air after erosion } \\ 0 \text { for } \mathbf{r} \in \text { regions with uncertain material and regions that are not of interest }\end{array}\right.$

Non-zero values in $w(\mathbf{r})$ can be used to emphasise the importance of any material or air.

It is common for many of today's users of industrial $\mathrm{XCT}$ devices to use empirical correction functions that try to compensate for disturbing effects of beam hardening and scattered radiation. This even applies for multi-material parts, e.g. by using predefined correction functions for linearisation that are provided by manufacturers of XCT devices and are, in theory, able to compensate for non-linear effects in monomaterial scenarios. Results of Tan et al. [14] have shown that those predefined correction curves should be used carefully, since they can induce additional measurement errors. This work studies whether the proposed adaption of ECC to multi-materials is adequate for dimensional metrology.

When considering only the linearisation aspect, the basic idea of the second correction technique, EDEC by Kachelrieß et al. [4], is very similar to the ECC method. The main difference is that EDEC needs at least two full XCT scans with different X-ray spectra to perform the homogenisation, while the corrected attenuation values of Eq. (1) are instead calculated by

$$
\begin{aligned}
p_{\text {EDEC }} & =P\left(q_{1}, q_{2}\right)=\sum_{i=0}^{I} \sum_{k=0}^{K} c_{i k} P_{i k}\left(q_{1}, q_{2}\right) \\
& =\sum_{i=0}^{I} \sum_{k=0}^{K} c_{i k} q_{1}^{i} q_{2}^{k} \text { with } c_{00}=0
\end{aligned}
$$

with $q_{1}$ being the attenuation value of the low energy (LE) and $q_{2}$ that of the high energy (HE) projections. For practical and economic reasons, EDEC is used in this work as a dualenergy approach, although in general, it is a multi-energy approach. Furthermore, $P_{i k}\left(q_{1}, q_{2}\right)$ represents the $(I+1)(\mathrm{K}+$ 2)/2 different basis images that are, in contrast to ECC, a polynomial composition of two attenuation values (LE and HE). Note that within this study, only the beam hardening correcting aspect of EDEC is of interest and not the extraction of the effective atomic number and the density of the inspected materials.

\subsection{Description of the Multi-material Reference Standard Used}

Properties of the ECC and EDEC methods are investigated using the MM-HC shown in Fig. 1. It is designed to analyse the multi-material influence on the length measurement error ( $E$-test) in acceptance testing of XCT systems used for dimensional metrology [2]. The cube investigated within this work has a size of $30 \mathrm{~mm} \times 30 \mathrm{~mm} \times 30 \mathrm{~mm}$ and consists of two symmetric parts made of aluminium (EN AW 5083) and titanium (Ti6Al4V). This material combination represents a multi-material case where typical multi-material errors can be observed. Conversely, this combination does not reach the domain where the projection data is massively distorted as is the case in more challenging combinations (e.g. steel and plastics, tungsten and plastics, etc.). The cube features 17 holes used as measurement features as well as 12 "V"-shaped grooves, G1-G12, on the outside used to establish reproducible measurement positions, see Fig. $1 \mathrm{~b}$ and Fig. 1c. Additionally, the cube features a step-like "cut" shape enabling different multi-material ratios along the standard's height, see Fig. 1d. Different ratios between the materials are present at grooves G2-G6, which allows in- and inter-material measurands in a multi-material scenario at a

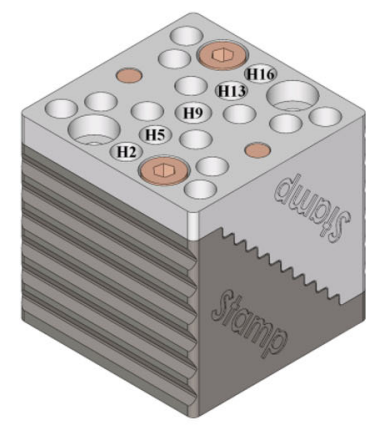

(a)

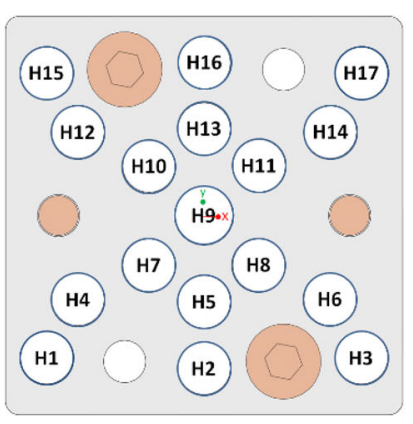

(b)

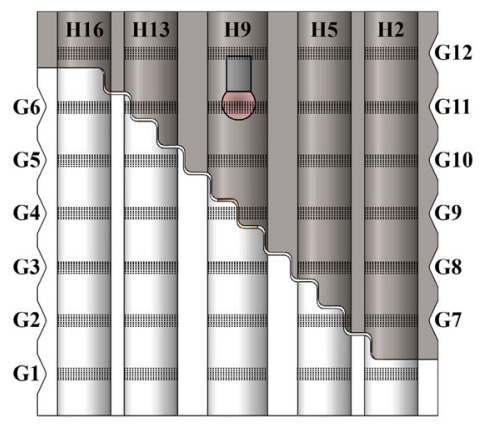

(c)

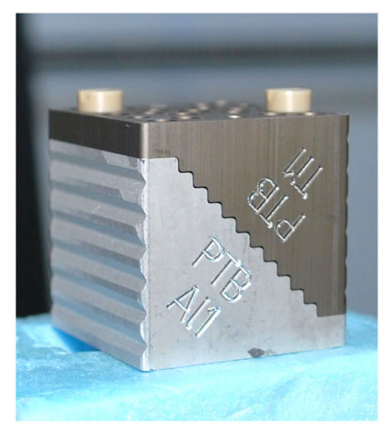

(d)

Fig. 1 a Multi-material hole cube (MM-HC); b top view of the MM-HC with labelling of bore holes in use; c central cut of the MM-HC highlighting the stepwise diagonal cut separating materials; d photograph showing the real XCT measurement setup (vertical CT rotary axis) 
horizontal setting. Measurands in mono-material scenarios are given at levels $\mathrm{G} 1(100 \% \mathrm{Al})$ and $\mathrm{G} 12(100 \% \mathrm{Ti})$.

\subsection{Experimental XCT Setup}

XCT scans of the MM-HC are performed with PTB's Nikon MCT 225 system (using Nikon Metrology X-Inspect 3.1.9 acquisition software) with a voxel size of approximately $(32 \mu \mathrm{m})^{3}$. It should be noted that the standard is scanned in horizontal orientation with a vertical rotation axis as shown in Fig. 1d to allow conclusions for different well-defined material ratios along the standard. Due to the circular scanning trajectory with a middle plane approximately at the height of groove G4, cone beam artefacts-especially at the top and bottom planar surfaces of the cube-would hinder measurements. Within this work however these plane surfaces are not of interest. Reconstructions are performed by filtered-back projection [15] without using the manufacturer's reconstruction software. Details on the evaluation procedure can be found in Sect. 2.4.

The goal of this study is to compare measurement results achieved with uncorrected and beam hardening corrected scans of the MM-HC for a total given scan time of $200 \mathrm{~min}$. The scan parameters of all XCT scans performed on the MM$\mathrm{HC}$ are shown in Table 1, where ME refers to medium energy, LE to low energy and HE to high energy. Varying energy levels of the spectra are achieved by using different combinations of X-ray tube voltages as well as prefilter materials and prefilter thicknesses. The ME parameters represent a typical parameter selection by an experienced XCT operator for a single energy scan of the MM-HC. The ECC correction scheme is applied to the ME scan data. Furthermore, the LE and HE scans are used for the dual-energy-based beam hardening correction (EDEC). They have a combined total scan time of $200 \mathrm{~min}$, i.e. the same time which is used for the single ME scan. The integration time of the HE scan is selected to be slightly longer to compensate for the intensity loss caused by the high amount of pre-filtering to harden the spectrum.

The X-ray tube voltages and prefilters of the LE and HE scan are selected to assure that they result in different artefacts in regions that should be represented by homogeneous grey values in the XCT images. The parameters are a trade-off between spectral separation and noise. X-ray tube power and thus the target current are selected with respect to the voxel size in order to reduce the geometrical blur of the focal spot.

Small residual scaling errors of the XCT scans are corrected by a multi-sphere scale correction to further improve the accuracy of the measurements additionally to the BHC methods and to allow an evaluation of the correction techniques free from other error sources. Each scale correction consists of a bracket of two scans of a multi-sphere reference standard. The scale correction is applied as the average
Table 1 Summary of scanning parameters for all performed XCT scans

\begin{tabular}{|c|c|c|c|c|}
\hline Parameter & Unit & ME scan & LE scan & HE scan \\
\hline $\begin{array}{l}\text { Acceleration } \\
\text { voltage }\end{array}$ & $\mathrm{kV}$ & 215 & 200 & 225 \\
\hline $\begin{array}{l}\text { Target } \\
\text { current }\end{array}$ & $\mu \mathrm{A}$ & 190 & 200 & 160 \\
\hline Tube power & W & 40.9 & 40 & 36 \\
\hline $\begin{array}{l}\text { Filter } \\
\text { material }\end{array}$ & & Sn $0.5 \mathrm{~mm}$ & $\mathrm{Cu} 0.5 \mathrm{~mm}$ & $\mathrm{Ag} 1.0 \mathrm{~mm}$ \\
\hline $\begin{array}{l}\text { Exposure } \\
\text { time }\end{array}$ & $\mathrm{ms}$ & 4000 & 2000 & 2829 \\
\hline $\begin{array}{l}\text { Detector } \\
\text { gain }\end{array}$ & $\mathrm{dB}$ & 12 & 12 & 24 \\
\hline $\begin{array}{l}\text { Number of } \\
\text { projec- } \\
\text { tions }\end{array}$ & & 3000 & 2490 & 2490 \\
\hline Scan time & $\min$ & 200 & 83 & 117 \\
\hline $\begin{array}{c}\text { Source- } \\
\text { object } \\
\text { distance }\end{array}$ & $\mathrm{mm}$ & 186.269 & & \\
\hline $\begin{array}{l}\text { Source- } \\
\text { detector } \\
\text { distance }\end{array}$ & $\mathrm{mm}$ & 1173.785 & & \\
\hline Voxel size & $\mu \mathrm{m}$ & 31.738 & & \\
\hline $\begin{array}{l}\text { Estimated } \\
\text { focal spot } \\
\text { diameter }\end{array}$ & $\mu \mathrm{m}$ & $20-22$ & & \\
\hline $\begin{array}{l}\text { Reconstruction } \\
\text { filter }\end{array}$ & & \multicolumn{3}{|c|}{ NR2 (Hanning) } \\
\hline
\end{tabular}

Parameters mentioned at the bottom are constant

of these two scans. Figure 2 shows flowcharts for the two series of measurements, one for the single energy ME scan and one for the dual-energy approach (LE+HE). For the ME and LE scan, the flat-field corrections (FFCs) have been performed at the beginning of both scan series. There have been no movements of axes during one series to allow a proper scaling correction. Scans for scale correction are done with scan parameters of the following or previous MM-HC scans, except that the number of projections is reduced to 2000 to speed up the process. The average of the scale correction values determined by the multi-sphere scans before and after scans of the MM-HC scans are used as correction values.

The thermal expansion of the cube materials has been neglected since the temperature in the XCT cabinet was recorded to be $(20.5 \pm 0.3){ }^{\circ} \mathrm{C}$. Thus, thermal expansion could lead to errors smaller than $0.3 \mu \mathrm{m}$ only. Temperatureinduced focal spot movements due to different operational settings (ME, LE and HE) of the X-ray tube are avoided by appropriate stabilisation times of at least $1.5 \mathrm{~h}$.

\subsection{Evaluation Procedure}

Dimensional length measurement error evaluations, i.e. deviation from the length reference values of tactile CMS 
Fig. 2 Flowcharts for the two series of experiments containing scans of the MM-HC and scale corrections with low, medium and high energies

Fig. 3 Multi-threshold approach applied in the evaluation of the MM-HC; a workflow step $3 \mathrm{a}$ where the $\mathrm{Al}$ threshold (red line) is customised for $\mathrm{Al}$; $\mathbf{b}$ slice of the resulting volume when using the threshold for $\mathrm{Al}$ (top half is $\mathrm{Al})$, white contours represent the surfaces determined; $\mathbf{c}$ workflow step $3 \mathrm{~d}$ where the $\mathrm{Ti}$ threshold (green line) value is customised for $\mathrm{Ti}$; $\mathbf{d}$ slice of the resulting volume when using the threshold for Ti (bottom half is Ti), white contours again represent the surfaces determined

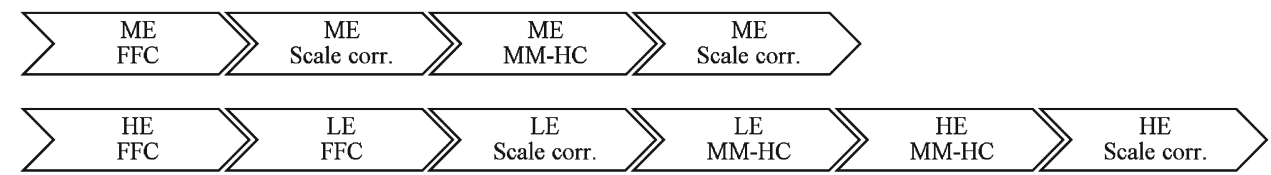

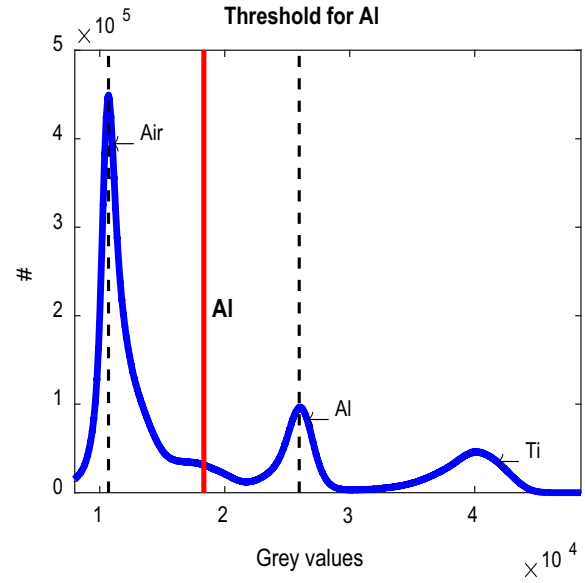

(a)

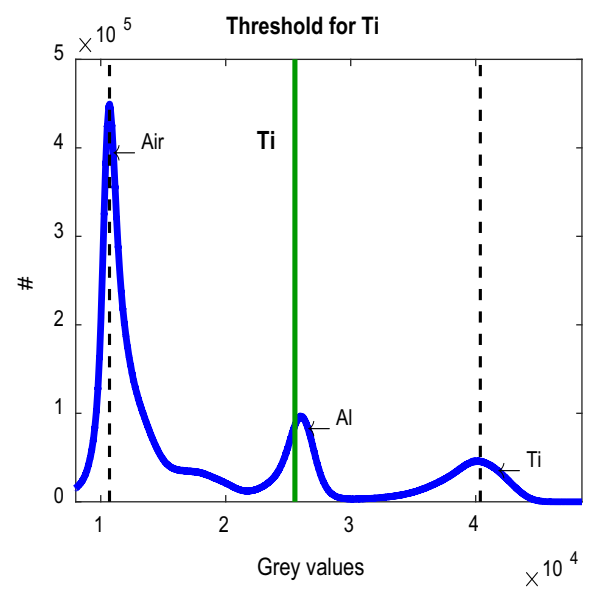

(c)

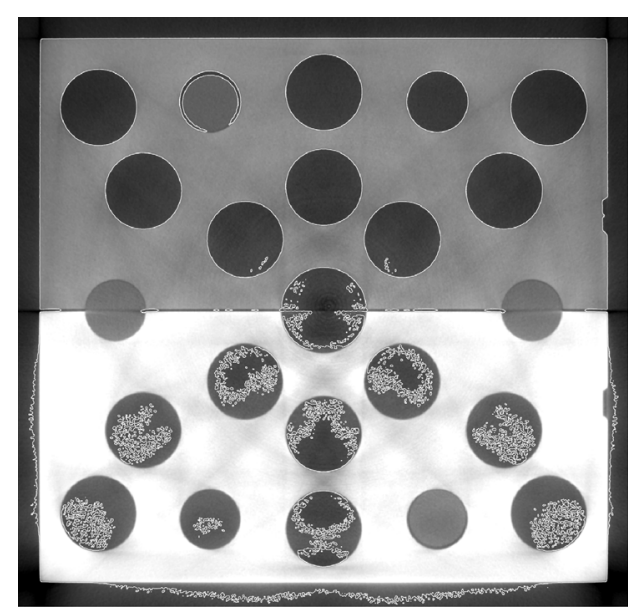

(b)

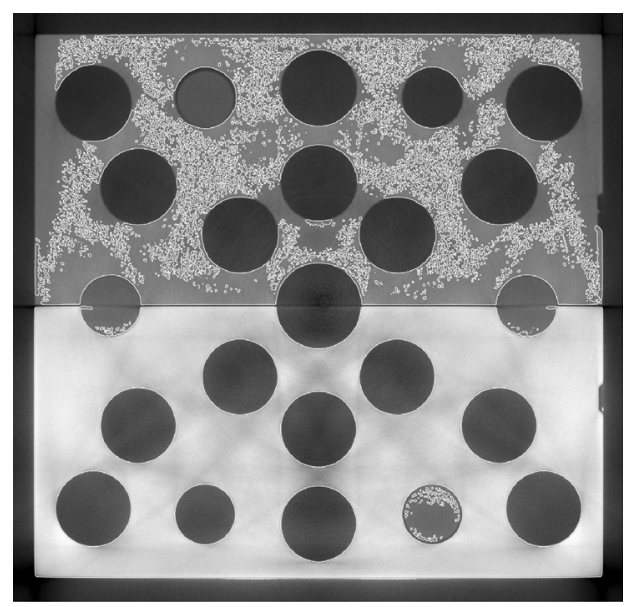

(d) measurements, were performed to investigate the measurement results achieved with uncorrected data as well as beam hardening corrected data of the MM-HC. The tactile reference measurements of the MM-HC were performed at PTB using a tactile CMS Carl Zeiss Prismo Ultra 7 with a $1 \mathrm{~mm}$ diameter coated diamond probe. The diamond probe was used to avoid contamination of the probe sphere, especially when using ruby probes on aluminium surfaces. The maximum permissible error of the Carl Zeiss Prismo Ultra 7 for length measurementa according to ISO 10360-2:2009 is $0.6 \mu \mathrm{m}+\mathrm{L} / 500 \mu \mathrm{m}$ ( $\mathrm{L}$ in $\mathrm{mm}$ ); while for form deviation according to the VDI/VDE 2617 part 2.2, the maximum permissible error for roundness RONt is $0.6 \mu \mathrm{m}$.

The reference measurements are based on the measurements of all 17 holes at 7 heights indicated by the respective groove position. For each groove height, seven circumferen- tial lines at different heights (circumferential lines distance $25 \mu \mathrm{m}$ ) are measured inside the holes, see Fig. 1c. In total, about 500000 tactile CMS points have been assessed. The tactile data serves as references for the XCT scans performed on PTB's Nikon MCT 225 system evaluated with a measurement strategy similar to the calibration.

For multi-material assemblies with a large difference in the $\mathrm{X}$-rays attenuation, the standard procedure with a single threshold value for the method "advanced" surface determination of both materials does not provide acceptable results in the current version of the analysis software used (VG Studio Max 3.0.3). This is due to the fact that the starting value for the surface determination process does not consider two or more material peaks in the grey value histogram. When the peaks of the two materials are significantly apart from each other, as visible in Fig. 3a, there is a large offset for 
the initial threshold that is typically not compensated for by local threshold refinements of VG Studio Max 3.0.3. This is why an improved two-step surface determination approach was used for the evaluation of the MM-HC data. In the first step, the threshold value is customised for aluminium and in the second step for titanium, see Fig. 3. This approach is due to the large difference in the X-ray attenuation between $\mathrm{Al}$ and Ti. Slices of the resulting XCT volume (uncorrected ME) are shown in Fig. 3b and c, where the white contour represents the surface determined. The workflow of the analysis is described in [2] and below:

1. Reference measurement of the $\mathrm{HC}$ using a tactile $\mathrm{CMS} \rightarrow$ tactile CMS points

2. Calculation of reference lengths between tactile CMS points using different measurands (i.e. centre-to-centre lengths and bidirectional patch-based lengths) $\rightarrow$ tactile CMS reference length results (software GOM inspect Professional version 7.5 SR2)

3. XCT scans of the MM-HC (see Fig. 2) without and with subsequent $\mathrm{BHC}$

a. Surface determination optimised for $\mathrm{Al} \rightarrow \mathrm{XCT}$ surface $\mathrm{Al}$ (software VG Studio Max 3.0.3)

b. 3-2-1-based alignment procedure of the $\mathrm{HC}$ using only the half containing $\mathrm{Al}$ (more details of the registration procedure in [2]) $\rightarrow$ Aligned XCT data

c. CAD-based ROI of the Al part, dilation and extraction process of the Al-based $\mathrm{ROI} \rightarrow \mathrm{Al}$ volume (software VG Studio Max 3.0.3)

d. Surface determination optimised for $\mathrm{Ti} \rightarrow \mathrm{XCT}$ surface Ti (software VG Studio Max 3.0.3)

e. CAD-based ROI of the Ti part, dilation and extraction process of the Ti-based $\mathrm{ROI} \rightarrow \mathrm{Ti}$ volume (software VG Studio Max 3.0.3)

f. Load the tactile CMS points into the XCT surface(s) of the ROI volume from step $3 \mathrm{c}$ and $3 \mathrm{e}$ (XCT data aligned to calibration data) and assess corresponding XCT points $\rightarrow$ XCT points (two point clouds; one for $\mathrm{Al}$ and one for Ti) (software VG Studio Max 3.0.3)

g. Length measurements of the XCT points using different measurands (e.g. centre-to-centre lengths, bidirectional patch-based lengths) $\rightarrow$ XCT results (software GOM inspect Professional version 7.5 SR2)

h. Difference between XCT results and tactile CMS reference results is the measurement error (MATLAB)

The pre-processing approach is performed to remove wrong surface regions. A CAD model of one half of the $\mathrm{MM}-\mathrm{HC}$ is registered to the surface of one material in the $\mathrm{XCT}$ volume to create a region of interest (ROI) in the XCT volume. Then a dilation process is applied to this ROI to ensure that the entire surface along the holes is not cut and a small region of air around the material remains in this ROI. This pre-processing approach is applied to $\mathrm{Al}$ and Ti, respectively. Although the CAD model of the $\mathrm{HC}$ is used to create a CAD-based ROI in the XCT data, it has no metrological influence other than reducing the time of the fitting process of the tactile points to the XCT surface.

The dimensional analysis of the correction methods for the MM-HC is based on centre-centre length measurements as well as on bidirectional inner and outer length measurements. Centre-centre measurements are one special example of unidirectional (averaged) measurements and are evaluated as the distances between centre points of cylinders which have each been created by least-square fitting of a short cylinder (with $\sim 175 \mu \mathrm{m}$ height) at a specific height, see Fig. 4a. Bidirectional length measurements are evaluated as inner and outer length measurements. The distance is defined by two representative points created through dedicated patch operators, see Fig. 4b (outer measurement) and $4 \mathrm{c}$ (inner measurement). The patch operator used in this analysis decreases the noise of point measurements and allows a better analysis of the different beam hardening correction procedures. For the measurement analysed in this work, the patch operator covers a surface area of approx. $175 \mu \mathrm{m}^{2}$ and contains typically 300 calibration/measurement points.

For the centre-centre lengths, all possible distances between the holes are measured in the plane perpendicular to the hole axes. However, for the bidirectional measurements, at least six lengths (not independent) in seven main directions of the MM-HC are measured, see Fig. 4d. In total, 956 unidirectional centre-to-centre lengths and $2 \times 378$ bidirectional patch-based inner and outer lengths at different heights (i.e. different material ratios) were measured. The difference in the total number of analysed lengths is due to the calculation time which, for the patch-based analysis, is much longer than the one for centre-to-centre measurements. To provide a better view of the material influence depending on the material ratios, the average, the range and the standard deviation of length measurement errors per groove are evaluated for both centre-centre and bidirectional inner and outer length measurements.

\section{Results}

\subsection{Practical Aspects of the ECC and EDEC Approaches}

In practice, it is useful to perform the optimisation procedure of ECC and EDEC only on some selected slices, instead of using the reconstructed volume of the complete specimen. This reduces the calculation effort of the optimisation drastically. In the presented case, seven slice images, located at the grooves G1 (Al mono-material layer), G2- 


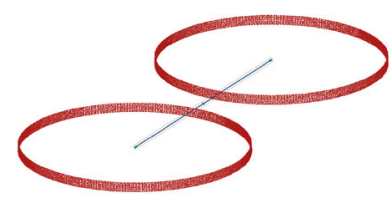

(a)

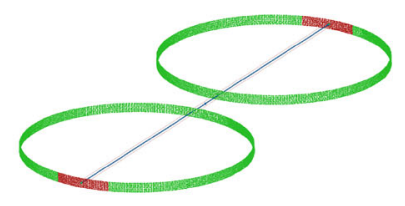

(b)

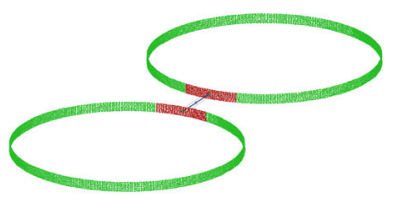

(c)

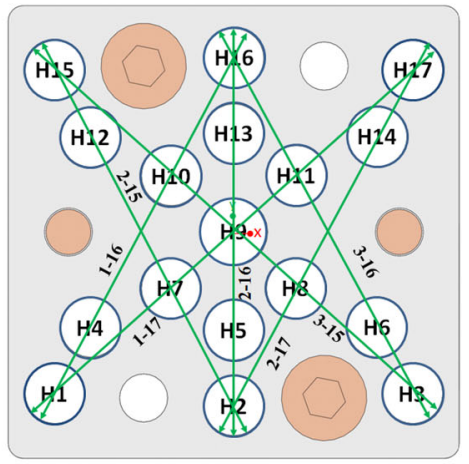

(d)

Fig. 4 Measurands for the evaluation of the MM-HC; red regions represent the patch (area/points) used for the creation of the length measurand: a centre-centre measurements (final elements are cylinder centres); $\mathbf{b}$ bidirectional inner measurements (final elements are representative points based on the patch); $\mathbf{c}$ bidirectional outer measurements

G6 (Al/Ti multi-material layers) and G12 (Ti mono-material layer), respectively, are used for the determination of coefficients $\mathrm{c}$. Therefore, also seven template $t(\mathbf{r})$ and weighting images $w(\mathbf{r})$ are created that define regions that should be homogenised.

The generation of templates was done in three steps. First, global thresholding (ISO-50\%) was used on uncorrected ME data for ECC templates and on HE data for EDEC. The thresholds from air to aluminium and air to titanium were calculated based on cumulated histograms that contained all seven slices. This initial binarisation led to over- and undersegmented regions where severe artefacts were present.

Therefore, in the second step, the initial binarisations were manually corrected by using regional growing methods and manual filling in combination with morphological operations (erosion, dilation, closing, opening) to refine the binary images. The sequence and parameters of the applied methods were adapted to the local appearance of the artefacts. It is of great importance to create binarisations and subsequently a weighted template $w(\mathbf{r}) t(\mathbf{r})$ that excludes uncertain regions, where the correspondence to a material or air is unclear, e.g. voxels affected by partial volume effects. On the contrary, regions close to those voxels (e.g. at outer surfaces of the specimen) should be included to get rid of beam hardening where it is often most significant. Simple thresholding methods without visual checks and corrections would especially cause template errors in regions that are most relevant for homogenisation. As an example, Fig. 5 shows a set of three binarisations $(M=2$ : air, $\mathrm{Al}, \mathrm{Ti})$ for one ME slice image at groove G4. It can be seen from Fig. 5b that local region weights of 0 were used to exclude non-relevant air regions far from the outer surface of the MM-HC as well as four fixing screws made of polyether ether ketone (PEEK) (cf. Figure 4d) from the optimisation.

(final elements are representative points based on the patch); and $\mathbf{d}$ seven main directions for the bidirectional measurements (image shows inner lengths only); observe the two PEEK (plastics) pins and two PEEK (plastics) screws used to assemble the two parts

In the third step, we switched to linear indexing of the slice images, while consecutively using the three binary images (e.g. Fig. 5b, c and d) to mask zero values and concatenate the voxel values of all slices into one large one-dimensional vector $f(\mathbf{r})$. Three images each filled with the material's template values are masked and concatenate similarly to generate the template vector $t(\mathbf{r})$. Furthermore, three images each filled with $1 / V_{M}$ material are masked and concatenate to generate the weighting vector $w(\mathbf{r})$ with $V_{M}$ being the total number of voxels that correspond to material $M$ in the $M$ binary images. The applied weighting makes Eq. (4) a minimisation of summed mean squared errors between template values and the corrected XCT image.

Within this work, only polynomial functions as basis functions for the optimisation were used. Another common basis function type are exponential functions, which at first seem to be best suitable due to the exponential terms in BeerLampert's law in general and for polychromatic X-rays in particular. Nevertheless, real X-ray acquisitions are affected by additional effects besides beam hardening, e.g. scattered radiation or geometrical blur, whereby polynomial functions typically result in smaller values of the optimisation metric $E$ with the same number of coefficients in use (Eqs. (1) and (7)) for most applications and especially for the MM-HC.

Preliminary tests for the polynomial degrees have shown that the most appropriate degree is 3, whereby 3 leads to slightly smaller $E$ values in comparison to a degree of 2, but to similar values when compared to $4^{\text {th }}$ order polynomials. Polynomials of degree 4 seem to over-parametrise the optimisation problem and are not advisable for the MM-HC on the XCT device in use in this study, since they lead to slight overcompensation of beam hardening artefacts especially at outer surfaces of the MM-HC. Conclusions were similar for the ECC and the EDEC correction techniques. 


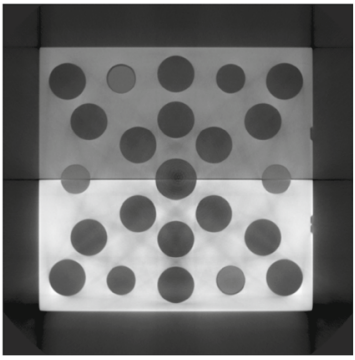

(a)

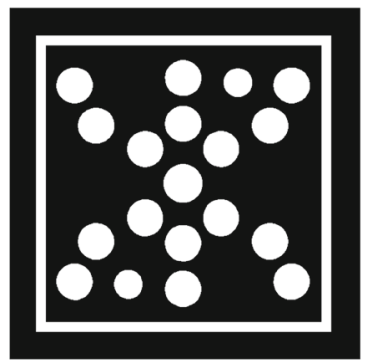

(b)

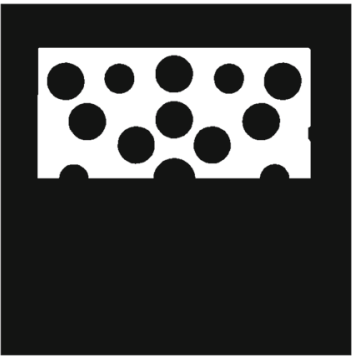

(c)

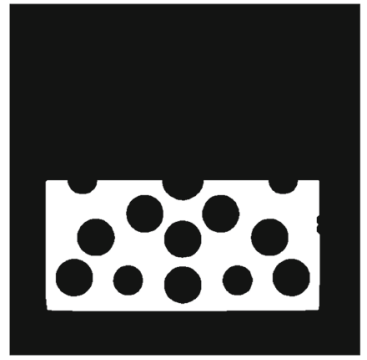

(d)

Fig. 5 Examples of slice images at groove G4 of a the uncorrected ME scan, with the binary images depicting the regions used for optimisation before the conversion into the weighted template $t(\mathbf{r}) w(\mathbf{r})$ for $\mathbf{b}$ air, $\mathbf{c}$ aluminium and $\mathbf{d}$ titanium shown as separate binary images

For ECC, a $3^{\text {rd }}$ order polynomial results in 3, and for EDEC, in 15 free parameters besides the fixed coefficients $c_{0}=0$ and $c_{00}=0$. Setting the first coefficients to 0 assures that the approximating function satisfies the boundary condition that the attenuation is zero in air, i.e. without any material to penetrate. The template values are calculated as the mean grey values within regions of aluminium and titanium that should be homogenised. For ECC, the seven representative uncorrected ME slices and for EDEC, the seven HE slices are used, which lead to

$t_{E C C}(\mathbf{r})=\left\{\begin{array}{r}\mu_{\mathrm{Al}}=0.0379 \text { for } \mathbf{r} \in \text { aluminium, } \\ \mu_{\mathrm{Ti}}=0.0744 \text { for } \mathbf{r} \in \text { titanium, } \\ 0 \text { for } \mathbf{r} \in \text { air },\end{array}\right.$

$t_{E D E C}(\mathbf{r})=\left\{\begin{array}{c}\mu_{\mathrm{Al}}=0.0326 \text { for } \mathbf{r} \in \text { aluminium, } \\ \mu_{\mathrm{Ti}}=0.0612 \text { for } \mathbf{r} \in \text { titanium, with } \mu \text { in } 1 / \mathrm{mm} . \\ 0 \text { for } \mathbf{r} \in \text { air, }\end{array}\right.$

The minimisation problem of Eq. (4) within this work is solved by a Nelder-Mead Simplex approach [16], which is an iterative heuristic method that is not based on gradients and is practical for the numerical optimisation of multi-dimensional problems. Note that this method does not guarantee results to be optimal or perfect. Different start values for the iteration have been tested to initialise $\boldsymbol{c}$ for the ECC and EDEC optimisation. The coefficients for ECC ( $3^{\text {rd }}$ order polynomial) shown in Table 2 have been determined reproducible for various start values, without actual proof for optimality. In contrast, the EDEC minimisation ended at significantly different coefficients for most of the tested initial $\boldsymbol{c}$ values, which might be caused by the large number of coefficients. This means that there are multiple solutions for the chosen stop criteria of the iteration. Two termination criteria had to be satisfied to stop the iteration: (i) a lower bound on the step size for the coefficients of $10^{-6}$ and a lower bound on the step size for the optimisation metric $E$ of $10^{-7}$. The highest grey value changes in the EDEC image by varying initial coefficients could be seen along the two outer lateral edges of titanium with approximately $\pm 5 \%$ of titanium's template value. In internal regions where the cube has measurement features, the changes were below $1 \%$. Therefore, this possible influence on dimensional measurements has not been further investigated for the EDEC procedure.

Table 2 shows the initial coefficients and initial search steps used to determine the final coefficients. The ECC search started with $p_{\mathrm{ECC}}=q_{1}$ and ended after 87 iterations. EDEC's search started at $p_{\mathrm{EDEC}}=0.5 q_{1}+0.5 q_{2}$ and was terminated after 1389 iterations. The final 3rd order polynomials of ECC and EDEC that satisfied the above-stated termination criterion are further evaluated regarding metrology in the following Sect. 3.3.

It can be noted that the coefficients of the beam hardening correction "preset 2" (e.g. used in [2]) provided by the system manufacturer (which are not used in this study) are close to the ECC result for a $2^{\text {nd }}$ order polynomial.

The ECC and EDEC methods are implemented as MATLAB/Octave scripts with additional use of VG Studio Max 3.0.5 for the generation and analysis of the weighted template.

\subsection{Image-Based Evaluation}

Figure 6 shows slice images for the comparison of the uncorrected ME scan at the top, the ECC corrected ME scan in the middle and the EDEC corrected (LE+HE) scan at the bottom for the groove heights G2, G4 and G6 from left to right. Both BHC methods are able to homogenise regions that are strongly affected by artefacts caused by beam hardening and scattered radiation, at all groove heights, which means for all $\mathrm{Al} / \mathrm{Ti}$ ratios, when compared to the uncorrected ME scan. In principle, the homogenisation is quantified by the optimisation metric, but the metric cannot be used to compare ECC and EDEC results. Therefore, contrast-to-noise ratio (CNR) values between air and aluminium as well as air and titanium are provided in Table 3. CNR values have been calculated using Eqs. (9) and (10) for all seven representative slices used for the optimisation. Template regions are utilised to calculate mean grey values $\mu$ and standard deviations $\sigma$. Note that 
Table 2 Presentation of the initial and final polynomial coefficients as well as the initial search steps for the iterative optimisation per exponent used in beam hardening corrections

\begin{tabular}{|c|c|c|c|c|c|}
\hline Data & $\begin{array}{l}\text { Initial search } \\
\text { steps }\end{array}$ & $\begin{array}{l}\text { Initial } \\
\text { coefficients }\end{array}$ & $\begin{array}{l}\text { Final } \\
\text { coefficients }\end{array}$ & Exponent of $q_{1}$ & Exponent of $q_{2}$ \\
\hline \multirow{2}{*}{$\begin{array}{l}\text { BH preset } 2 \\
\text { (manufacturer } \\
\text { setting) (2nd order } \\
\text { polynomial) }\end{array}$} & & & 0.5682 & 1 & \\
\hline & & & 0.1894 & 2 & \\
\hline \multirow{2}{*}{$\begin{array}{l}\text { ECC }(2 \text { nd order } \\
\text { polynomial })\end{array}$} & 0.1000 & 1 & 0.5642 & 1 & \\
\hline & 0.0563 & 0 & 0.2175 & 2 & \\
\hline \multirow{3}{*}{$\begin{array}{l}\text { ECC (3rd order } \\
\text { polynomial) }\end{array}$} & 0.1000 & 1 & 0.9578 & 1 & \\
\hline & 0.0563 & 0 & -0.3147 & 2 & \\
\hline & 0.0315 & 0 & 0.1752 & 3 & \\
\hline \multirow{15}{*}{$\begin{array}{l}\text { EDEC (3rd order } \\
2 \mathrm{~d}-\text { polynomial) }\end{array}$} & 0.1000 & 0.5 & 0.0804 & 1 & 0 \\
\hline & 0.1612 & 0.5 & 0.6914 & 0 & 1 \\
\hline & 0.0678 & 0 & -0.2270 & 1 & 1 \\
\hline & 0.0416 & 0 & -0.0179 & 2 & 0 \\
\hline & 0.1104 & 0 & 0.5307 & 0 & 2 \\
\hline & 0.0282 & 0 & 0.0272 & 2 & 1 \\
\hline & 0.0462 & 0 & -0.1722 & 1 & 2 \\
\hline & 0.0188 & 0 & -0.1222 & 2 & 2 \\
\hline & 0.0172 & 0 & -0.0125 & 3 & 0 \\
\hline & 0.0755 & 0 & 0.2257 & 0 & 3 \\
\hline & 0.0115 & 0 & 0.0623 & 3 & 1 \\
\hline & 0.0309 & 0 & 0.0943 & 1 & 3 \\
\hline & 0.0075 & 0 & -0.0310 & 3 & 2 \\
\hline & 0.0123 & 0 & 0.02660 & 2 & 3 \\
\hline & 0.0049 & 0 & 0.00233 & 3 & 3 \\
\hline
\end{tabular}

For ECC, $q_{1}$ are attenuation values of ME data. For EDEC, $q_{1}$ are attenuation values of LE and $q_{2}$ of $\mathrm{HE}$ data

Table 3 Mean grey values $\mu$, standard deviations $\sigma$ and CNR values calculated for all seven slices of the optimisation

\begin{tabular}{lllllrr}
\hline Data & $\mu_{\mathrm{Ti}}$ & $\sigma_{\mathrm{Ti}}$ & $\mu_{\mathrm{Al}}$ & $\sigma_{\mathrm{Al}}$ & $\mathrm{CNR}_{\mathrm{Ti}, \text { air }}$ & $\mathrm{CNR}_{\mathrm{Al}, \mathrm{air}}$ \\
\hline Uncorrected (ME) & 0.07410 & 0.00768 & 0.03762 & 0.00348 & 7.9 & 6.9 \\
ECC (ME) & 0.07485 & 0.00598 & 0.03147 & 0.00267 & 11.2 & 8.8 \\
EDEC (LE and HE) & 0.05715 & 0.01283 & 0.02994 & 0.00711 & 3.9 & 3.1 \\
\hline
\end{tabular}

Template regions define regions of air, $\mathrm{Al}$ and Ti. The calculation was performed on a common volume of all seven slices

$\sigma_{\mathrm{Al}}$ and $\sigma_{\mathrm{Ti}}$ were used in the denominator to quantify the homogeneity and noise of the two materials [17]. All seven slices and template regions are treated as one volume.

$\begin{aligned} \mathrm{CNR}_{\mathrm{Al}, \mathrm{air}} & =\frac{\mu_{\mathrm{Al}}-\mu_{\mathrm{air}}}{\sigma_{\mathrm{Al}}} \\ \mathrm{CNR}_{\mathrm{Ti}, \mathrm{air}} & =\frac{\mu_{\mathrm{Ti}}-\mu_{\mathrm{air}}}{\sigma_{\mathrm{Ti}}}\end{aligned}$

Table 3 depicts an increase in CNR from uncorrected to ECC corrected data, whereby $\mathrm{CNR}_{\mathrm{Ti}}$,air might be slightly higher than $\mathrm{CNR}_{\mathrm{Al} \text {,air }}$ since, during the ECC optimisation, the squared errors for the optimisation metric $E$ (see Eq. (4)) in titanium regions are larger and thus more relevant than those of aluminium. This might put more weight on the optimisation of titanium. Hence, ECC reduced artefacts caused by any kind of non-linear behaviour, which is confirmed by CNR values in Table 3 and images of Fig. 6. CNR values of EDEC corrected data are overall the lowest (i.e. worst). This is due to slightly smaller contrast terms in the enumerator of EDEC data in combination with denominators $\sigma_{\mathrm{Ti}}$ and $\sigma_{\mathrm{Al}}$ that are at least twice as high as standard deviations of uncorrected and ECC data. Furthermore, visual compar- 


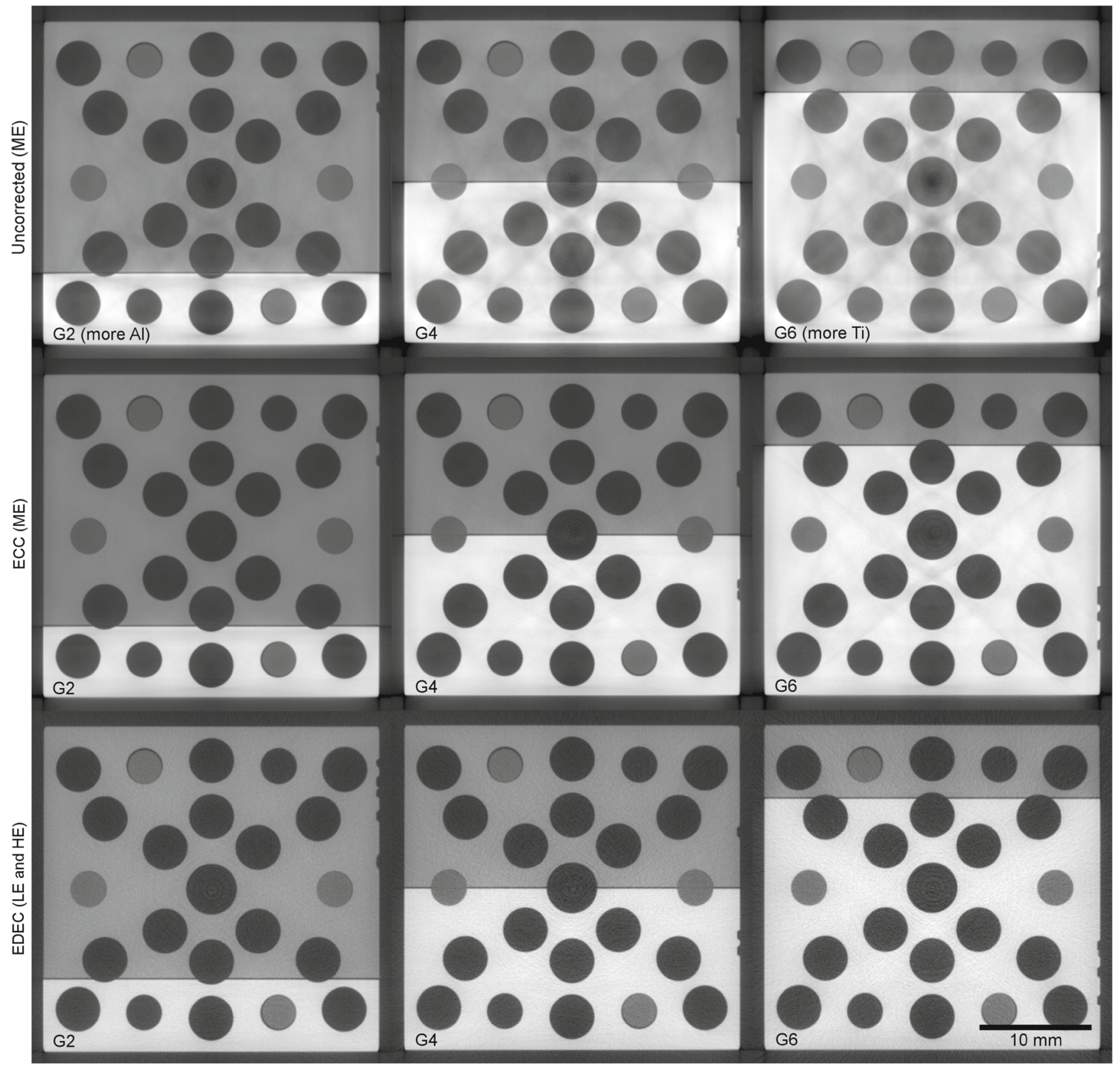

Fig. 6 Example of slice image of top row the uncorrected ME scan, middle row ECC corrected ME scan and bottom row EDEC corrected LE plus HE scan for the groove heights left column G2, middle column

isons in Fig. 6 depict that EDEC was able to compensate for artefacts even more than ECC, but is prone to noise and ring artefacts, which severely affected the CNR values. Possible influences of these findings on material transitions and thus dimensional measurements are discussed in the next section.

\subsection{Metrology Results}

Computed tomography datasets of the multi-material hole cube standards were obtained with the PTB's Nikon MCT
G4 and right column G6. Slices of uncorrected ME and ECC corrected ME were generated using a grey value windowing of $[-0.1,0.09]$ and the EDEC images with [-0.1, 0.075]

225 with different measurement parameters in order to compare the metrological performance of the selected BHC methods. An ME scan representing a typical set of scan parameters used by an experienced XCT user as well as an LE scan and an HE scan providing enough difference in the artefacts in the XCT image to apply the dual-energy-based EDEC correction were carried out.

Unidirectional (averaged) centre-centre measurements as well as bidirectional inner and outer measurements (cf. Figure 3) were performed and are presented in Fig. 7. To provide an overview of the measurement results, box plots with aver- 


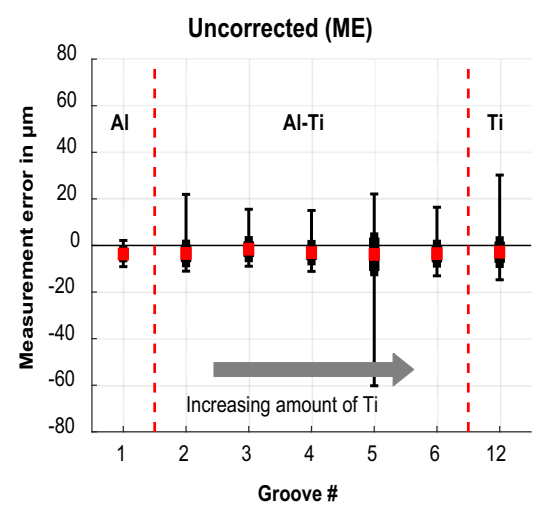

(a)

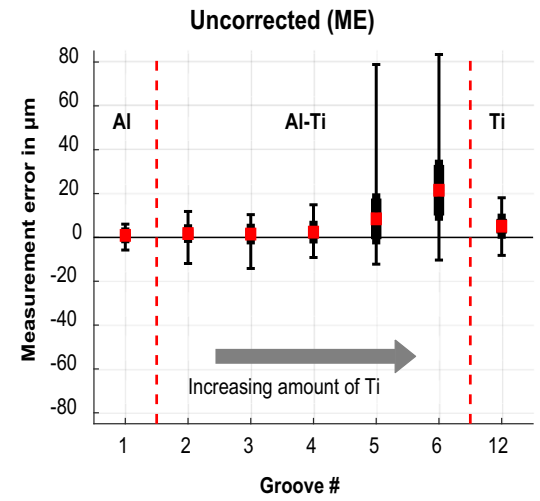

(d)

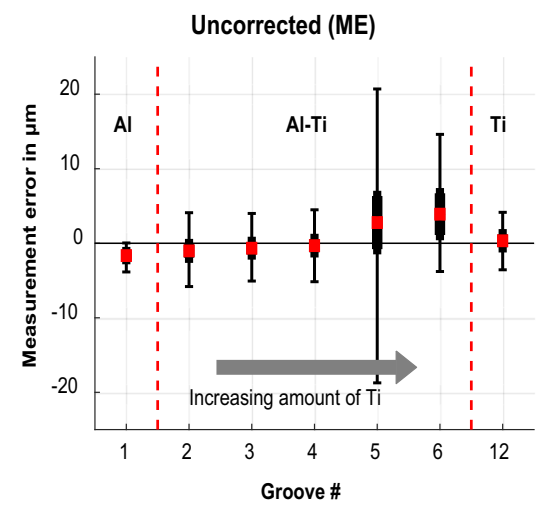

(g)

\section{Inner bidirectional measurements}

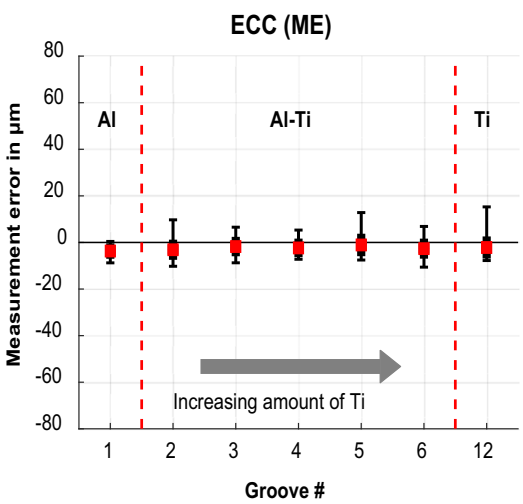

(b)

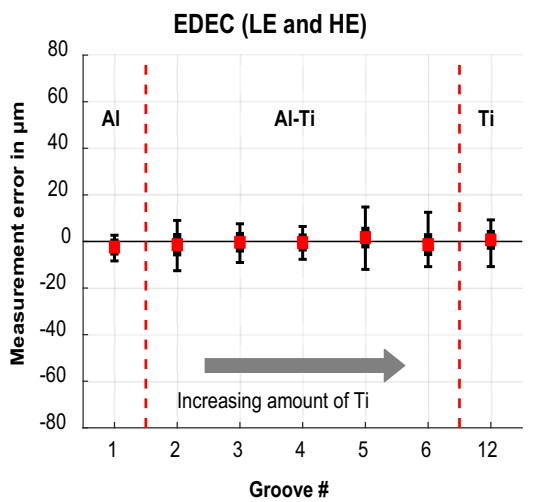

(c)

\section{Outer bidirectional measurements}

ECC (ME)

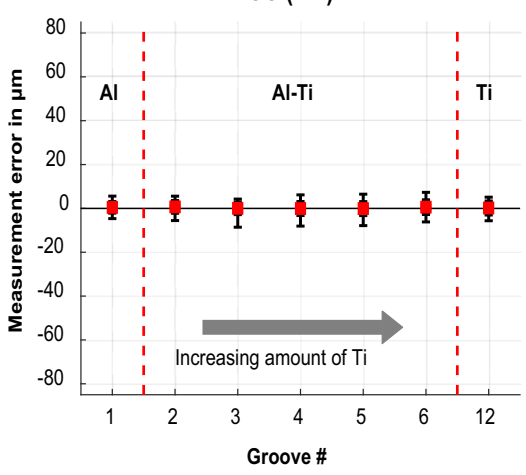

(e)

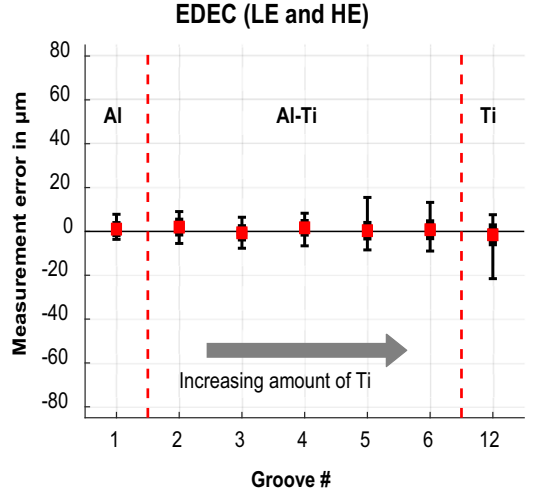

(f)

\section{Centre-centre unidirectional measurements}

ECC (ME)

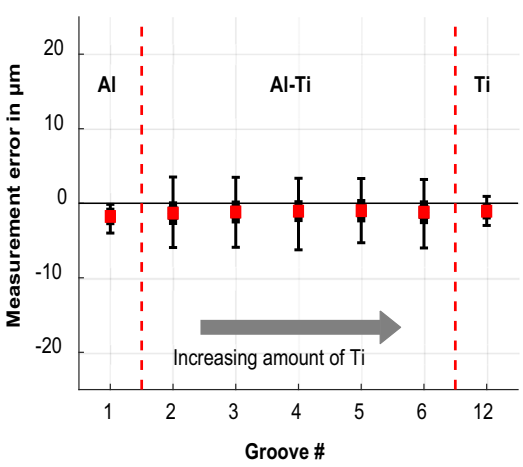

(h)

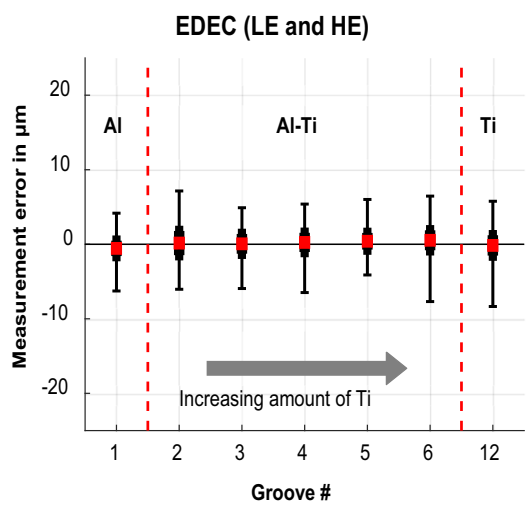

(i)

Fig. 7 Boxplots of performed bidirectional inner and outer measurements as well as centre-centre measurements (cf. Figure 4) for left column uncorrected, middle column ECC and right column EDEC data; voxel size $\approx 31.738 \mu \mathrm{m}$

age, standard deviation and maximum and minimum values per groove are given.

In general, a significant improvement of the results for both BHC methods could be observed, whereby the ECC method seems to give somewhat better results than the EDEC approach. Improvements are noticeable as smaller mean, minimum and maximum values at all grooves, but are especially strong at grooves G5 and G6 featuring a high Ti percentage.

For inner bidirectional measurements, a total error range of $75 \mu \mathrm{m}$ (i.e. approx. $2.3 \times$ voxel size) was observed for the uncorrected data, see Fig. 7a. While applying the correction 


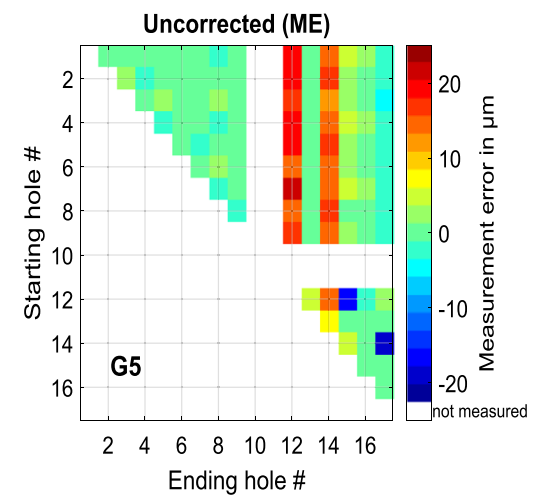

(a)

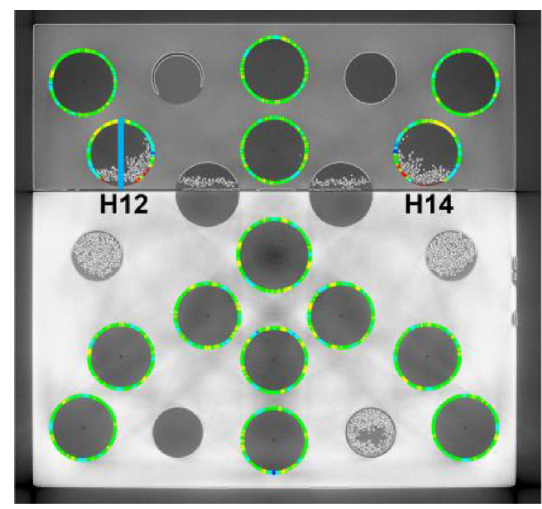

(d)

\section{Centre-centre unidirectional measurements}

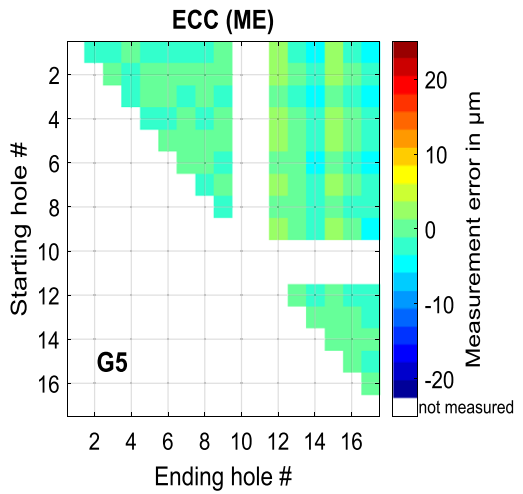

(b)

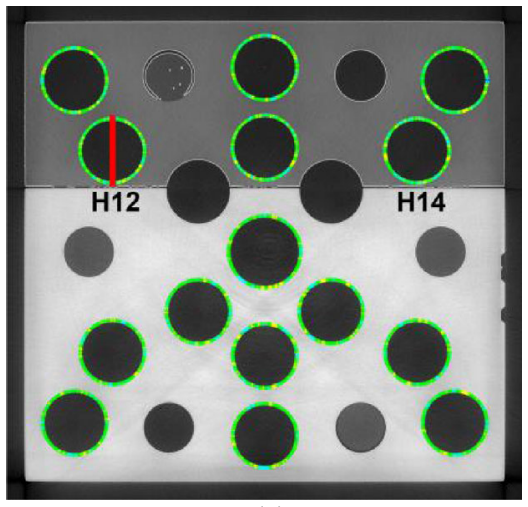

(e)

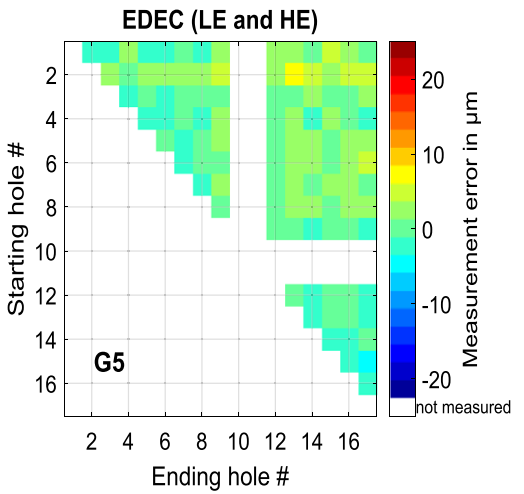

(c)

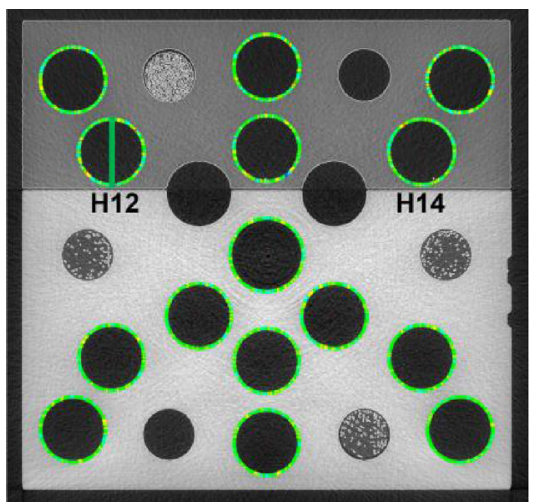

(f)

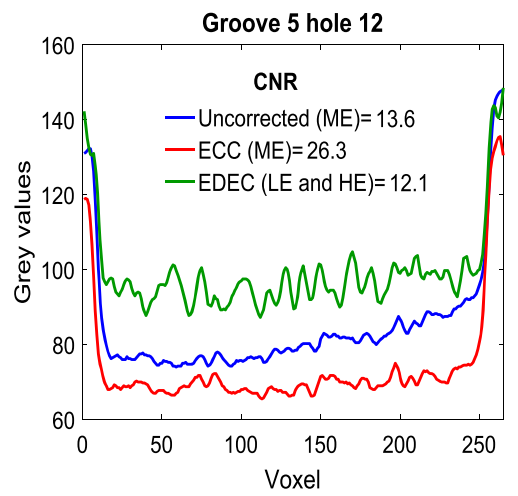

(g)

Fig. 8 Detailed analyses of measurements at groove G5 by a-c colour plots for all centre-to-centre measurements performed between the holes; d-f slice images at G5 (top part is Al; observe that artefact structures inside holes are not detected as surface points of the holes; this is due to a filtering effect of the surface point fitting procedure which enters into the final cylinder fit) and $\mathbf{g}$ grey value profiles through hole 12 at G5

around $15 \mu \mathrm{m}$ (i.e. $0.4 \times$ voxel size), while for the EDEC approach, the total range was bigger with $38 \mu \mathrm{m}$ (i.e. $1.2 \times$ voxel size), see Fig. 7e and $f$.

An even better improvement of the measurement results was also observed for the centre-centre unidirectional measurements. The uncorrected data presented a $39 \mu \mathrm{m}$ (i.e. $1.2 \times$ voxel size) range of the measurement error, see Fig. $7 \mathrm{~g}$. 
Table 4 Overview of the achieved total range of measurement errors covering $\mathrm{Al}, \mathrm{Al}-\mathrm{Ti}$ and Ti domains; voxel size $\approx 32 \mu \mathrm{m}$

\begin{tabular}{llclccc}
\hline & Uncorr. & ECC & EDEC & Uncorr. & ECC & EDEC \\
\hline Length measurement & & Given in $\mu \mathrm{m}$ & \multicolumn{3}{c}{ As multiple of the voxelsize } \\
Centre-centre & 39 & 9 & 16 & 1.2 & 0.3 & 0.5 \\
Bidirectional inner & 75 & 28 & 35 & 2.4 & 0.9 & 1.1 \\
Bidirectional outer & 98 & 15 & 38 & 3.0 & 0.5 & 1.2 \\
\hline
\end{tabular}

the total range of measurement errors. The mean errors are comparable between the ECC and EDEC approaches, which indicates that the material influence is reduced for both $\mathrm{BHC}$ methods.

Although, minimising temperature-related geometrical misalignments and scaling errors between the LE and HE scans was tried, some influences were still noticed. Prior to applying the scaling correction, the relative stability between the scaling correction scans before and after the actual scans was less than $0.3 \mu \mathrm{m}$ for the ME scan series and $3.0 \mu \mathrm{m}$ for the LE+HE scan series over a length of $40 \mathrm{~mm}$. This indicates that the LE and HE scans had slightly different magnifications. One additional cause worth noting is the $\mathrm{X}$ ray spectra-dependent magnifications that have been reported by Illemann et al. [18].

\section{Conclusions}

The two beam hardening correction techniques, EDEC and ECC, were successfully applied to an industrial multimaterial scenario, with materials that show a significant difference in their absorption properties. The test scenario was created using a calibrated multi-material hole cube made of aluminium and titanium originally designed for developing future multi-material acceptance testing. For ECC, originally designed for mono-material cases, modifications were proposed and implemented to enable the method to also be used for multi-material scenarios. Even though the procedures under study do not need any preliminary knowledge of the specimen (geometry, position and materials), a manual refinement of the template images for both procedures was necessary. This procedure, mainly working with morphological operations, ensures optimised conditions for the reduction of artefacts caused by beam hardening and partially scattered radiation, leading to more reliable measurements by minimising measurement errors.

Table 4 shows a summary of the achieved improvements in terms of total ranges of measurement errors for all measurements on uncorrected, ECC and EDEC corrected data.

Both BHC approaches show significant improvements for the dimensional measurands under study (centre-centre length measurement errors and as well bidirectional inner and outer length measurement errors), especially for the highest errors which occur at low absorbing aluminium areas in mea- 
surement slices with a high content of the strong absorbing titanium. The improvement in the total range of measurement errors reaches a factor between 2.1 (for the EDEC correction of bidirectional inner lengths) and 4.3 (for the ECC correction of centre-centre lengths).

Only on rare occasions-slices with $100 \%$ titanium and analysing centre-centre lengths only-does the EDEC correction result in larger outliers compared to the uncorrected state mainly due to noise, even though the remaining inhomogeneities related to artefacts and mean measurement errors are smaller compared to ECC.

For the experimental setup used to scan the multi-material hole cube, the use of an additional spectrum does not seem to be beneficial for a constant total scan time of $200 \mathrm{~min}$. ECC made more efficient use of the total exposure time and led to even better corrections. It is also easier for operators of XCT devices to handle on a daily basis, since no special care is needed to assure minimal misalignments between the LE and the HE scans and only one FFC needs to be assessed for the ME scan. It has to be mentioned that the potential for reducing beam hardening artefacts with ECC potentially worsens, the more different the attenuation behaviour of scanned materials is, since this linearisation method is actually only capable of dealing with mono-material scenarios. ECC may not be able to handle even larger absorption differences properly and thus EDEC is expected to outperform ECC in more challenging cases with extreme variations in the attenuation behaviour of investigated materials (steel and plastics, tungsten and plastics etc.).

All in all, both correction techniques may help to improve the measurement capabilities of recent XCT systems when measuring multi-materials on a level that today's user expects from mono-material measurements. At the moment both applied procedures require manual adjustment—a step which the authors believe could be automated using rule-based refinement procedures and preliminary knowledge.

Acknowledgements Open access funding provided by University of Applied Sciences Upper Austria. This paper is a collaborative work between the University of Applied Sciences Upper Austria and the Physikalisch-Technische Bundesanstalt PTB (Germany) and is supported by the "K-Project for non-destructive testing and tomography plus" financed by the Österreichische Forschungsförderungsgesellschaft (FFG) (Grant Number 843540) and the governments of Upper Austria and Styria as well as the EU Marie Curie project "International Network for the Training of Early stage Researchers on Advanced Quality control by Computed Tomography" (INTERAQCT) (FP7-PEOPLE-2013-ITN, Grant Number 607817).

Open Access This article is distributed under the terms of the Creative Commons Attribution 4.0 International License (http://creativecomm ons.org/licenses/by/4.0/), which permits unrestricted use, distribution, and reproduction in any medium, provided you give appropriate credit to the original author(s) and the source, provide a link to the Creative Commons license, and indicate if changes were made.

\section{References}

1. Grimmer, R., Kachelrieß, M.: Selbstkalibrierende Computertomografie. In: Proceedings of the 3rd Conference on Industrial Computed Tomography (iCT2010), Wels, Austria (2010)

2. Borges de Oliveira, F, Bartscher, M., Neuschaefer-Rube, U., Tutsch, R., Hiller, J.: Creating a multi-material length measurement error test for the acceptance testing of dimensional computed tomography systems. In: Proceedings of the 7th Conference Industrial Computed Tomography (iCT2017), Leuven, Belgium (2017)

3. Kachelrieß, M., Sourbelle, K., Kalender, W.A.: Empirical cupping correction: a first-order raw data precorrection for cone-beam computed tomography. Med. Phys. 33(5), 1269-1274 (2006). https:// doi.org/10.1118/1.2188076

4. Kachelrieß, M., Berkus, T., Stenner, P., Kalender, W.A.: Empirical Dual Energy Calibration (EDEC) for cone-beam computed tomography. In: 2006 IEEE Nuclear Science Symposium Conference Record, vol. 4, pp. 2546-2550 (2006). https://doi.org/10. 1109/nssmic.2006.354428

5. Stenner, P., Berkus, T., Kachelrieß, M.: Empirical dual energy calibration (EDEC) for cone-beam computed tomography. Med. Phys. 34(9), 3630-3641 (2007). https://doi.org/10.1118/1.2769104

6. Krumm, M., Kasperl, S., Franz, M.: Reducing non-linear artifacts of multi-material objects in industrial $3 \mathrm{~d}$ computed tomography. NDT and E Int. 41(4), 242-251 (2008). https://doi.org/10.1016/j. ndteint.2007.12.001

7. Kyriakou, Y., Meyer, E., Prell, D., Kachelrieß, M.: Empirical beam hardening correction (EBHC) for CT. Med. Phys. 37(10), 5179-5187 (2010). https://doi.org/10.1118/1.3477088

8. Maaß, C., Meyer, E., Kachelrieß, M.: Exact dual energy material decomposition from inconsistent rays (MDIR). Med. Phys. 38(2), 691-700 (2011). https://doi.org/10.1118/1.3533686

9. Yang, Q., Maass, N., Tian, M., Elter, M., Schasiepen, I., Maier, A.K., Hornegger, J.: Multi-material beam hardening correction (MMBHC) in computed tomography. In: Proceedings of the 12th International Meeting on Fully Three-Dimensional Image Reconstruction in Radiology and Nuclear Medicine (2013)

10. Man, B.D., Nuyts, J., Dupont, P., Marchal, G., Suetens, P.: An iterative maximum-likelihood polychromatic algorithm for ct. IEEE Trans. Med. Imaging 20(10), 999-1008 (2001). https://doi.org/10. 1109/42.959297

11. Brabant, L., Pauwels, E., Dierick, M., Loo, D.V., Boone, M., Hoorebeke, L.V.: A novel beam hardening correction method requiring no prior knowledge, incorporated in an iterative reconstruction algorithm. NDT and E Int. 51(Supplement C), 68-73 (2012). https:// doi.org/10.1016/j.ndteint.2012.07.002

12. Zhao, Y., Li, M.: Iterative beam hardening correction for multimaterial objects. PLoS ONE 10(12), 607 (2015)

13. Heinzl, C., Kastner, J., Gröller, E.: Surface extraction from multimaterial components for metrology using dual energy CT. IEEE Trans. Vis. Comput. Gr. 13(6), 1520-1527 (2007). https://doi.org/ 10.1109/TVCG.2007.70598

14. Tan, Y., Kiekens, K., Welkenhuyzen, F., Kruth, J., Dewulf, W.: Beam hardening correction and its influence on the measurement accuracy and repeatability for CT dimensional metrology applications. In: Proceedings of the 4th Conference on Industrial Computed Tomography (iCT2012), Wels, Austria (2012)

15. Feldkamp, L.A., Davis, L.C., Kress, J.W.: Practical cone-beam algorithm. J. Opt. Soc. Am. A 1(6), 612-619 (1984). https://doi. org/10.1364/josaa.1.000612

16. Lagarias, J.C., Reeds, J.A., Wright, M.H., Wright, P.E.: Convergence properties of the nelder-mead simplex method in low dimensions. SIAM J. Opt. 9(1), 112-147 (1998). https://doi.org/ $10.1137 / \mathrm{S} 1052623496303470$ 
17. Reiter, M., Krumm, M., Kasperl, S., Kuhn, C., Erler, E., Weiß, D., Heinzl, C., Gusenbauer, C., Kastner, J.: Evaluation of transmission based image quality optimisation for X-ray computed tomography. In: Proceedings of the 4th Conference on Industrial Computed Tomography (iCT2012), Wels, Austria (2012)
18. Illemann, J., Bartscher, M.: X-ray spectrum dependence of the magnification of cone-beam CT. In: Proceedings of the 7th Conference on Industrial Computed Tomography (iCT2017), Leuven, Belgium (2017) 